\title{
Effects of Extended Cocaine Access and Cocaine Withdrawal on Choice Between Cocaine and Food in Rhesus Monkeys
}

\author{
Matthew L Banks' and S Stevens Negus*, 1,2 \\ 'Department of Pharmacology and Toxicology, Virginia Commonwealth University, Richmond, VA, USA; ${ }^{2}$ Alcohol and Drug Abuse Research \\ Center, Harvard Medical School, McLean Hospital, Belmont, MA, USA
}

\begin{abstract}
Chronic drug use may lead to sufficient drug intake to produce dependence and the emergence of abstinence signs during withdrawal. Although withdrawal can increase the reinforcing effects of some drugs (eg opioids), the impact of withdrawal on the reinforcing effects of stimulants like cocaine is less clear. This study used a novel cocaine vs food choice procedure to examine the relative reinforcing strength of cocaine before, during, and after exposure to graded levels of extended cocaine access. Responding in four rhesus monkeys was maintained by cocaine $(0-0.1 \mathrm{mg} / \mathrm{kg} /$ injection) and food delivery under a concurrent-choice schedule during daily 2-h sessions. Under baseline conditions, cocaine maintained a dose-dependent increase in cocaine choice. Subsequently, subjects were exposed to and withdrawn from periods of extended cocaine access, which was accomplished by implementing daily 21 -h supplemental sessions of cocaine self-administration in addition to daily choice sessions. During supplemental sessions, cocaine $(0.1 \mathrm{mg} / \mathrm{kg} / \mathrm{injection})$ was available under a fixed-ratio 10/time-out $X$ schedule, and the duration of the time-out was varied from 30 to 7.5 min. Cocaine intake increased 10 -fold to $>11 \mathrm{mg} / \mathrm{kg} /$ day during exposure to supplemental sessions with the shortest post-injection time-out. However, parameters of cocaine choice were not significantly affected either during or after extended cocaine access. These results do not support the hypothesis that cocaine withdrawal increases the reinforcing strength of cocaine. This differs from results with the opioid agonist heroin and suggests that withdrawal may have different functions in the maintenance of opioid and stimulant abuse. Neuropsychopharmacology (2010) 35, 493-504; doi:I0.1038/npp.2009. I54; published online 23 September 2009
\end{abstract}

Keywords: cocaine; choice procedure; nonhuman primates; dependence; withdrawal; alternative reinforcer

\section{INTRODUCTION}

Chronic drug exposure can produce a dependent state as indicated by the emergence of abstinence signs during drug withdrawal. The particular constellation of abstinence signs associated with drug withdrawal varies by drug class and may include an increase in the reinforcing effects of drugs within that class in drug self-administration assays. For example, withdrawal in opioid-dependent subjects produces characteristic somatic signs, as well as an increase in the reinforcing effects of opioid agonists as determined using progressive-ratio and concurrent-choice schedules of reinforcement (Griffiths et al, 1975; Yanagita, 1978; Carrera et al, 1999; Negus, 2006; Negus and Rice, 2009). Insofar as reinforcing effects are predictive of abuse liability, these findings suggest that chronic drug exposure leading to dependence may enhance the abuse liability of some drugs and may contribute to the prevalence of addiction.

\footnotetext{
*Correspondence: Dr SS Negus, Department of Pharmacology and Toxicology, Virginia Commonwealth University, 410 North I2th Street, PO Box 980613, Richmond, VA 23298, USA, Tel: + I 8048283158 , Fax: + I804828 2117, E-mail: ssnegus@vcu.edu

Received 2 July 2009; revised 25 August 2009; accepted 26 August 2009
}

Moreover, these findings suggest that useful treatments might target mechanisms that underlie dependence- and withdrawal-associated increases in drug reinforcement. For example, we have shown earlier that methadone and other mu-opioid agonists block withdrawal-associated increases in heroin self-administration, and we have suggested that this effect may contribute to the clinical utility of agonist medications for the treatment of opioid dependence (Negus, 2006; Negus and Rice, 2009). In view of these considerations, there may be value in assessing the conditions under which dependence and withdrawal enhance the reinforcing effects of drugs in other drug classes.

Central nervous system stimulants such as cocaine have high abuse liability and maintain problematic levels of abuse. There are currently no FDA-approved medications for the treatment of cocaine abuse (Vocci et al, 2005), and medications development might benefit from evaluation of the magnitude and mechanisms of withdrawal-associated changes in cocaine reinforcement. It is now well established that withdrawal from chronic cocaine produces a characteristic set of abstinence signs, although these signs present more subtly than those produced by withdrawal from other abused drugs, such as opioids. For example, Gawin and Kleber (1986) described cocaine withdrawal as a 
'fluctuating clinical syndrome' with symptoms including anhedonia, anxiety, and irritability. Moreover, cocaine abstinence signs and symptoms can be objectively and reliably assessed using the cocaine selective severity assessment (Kampman et al, 1998). In preclinical studies, withdrawal from treatment with cocaine doses up to $60 \mathrm{mg} / \mathrm{kg} /$ day for 7 days in rats elicited somatic abstinence signs such as tremors, teeth chatters, and head shakes (Malin et al, 2000). Furthermore, withdrawal from either contingent or noncontingent extended cocaine exposure produces affective withdrawal signs including anxiogeniclike effects (Basso et al, 1999) and decreases in rates of food-maintained responding (Carroll and Lac, 1987; Kleven and Woolverton, 1991) and intracranial self-stimulation (Markou and Koob, 1991; Kenny et al, 2003). Finally, these behavioral studies are supported by neurochemical evidence during cocaine withdrawal showing decreased central nervous system glucose metabolism (Hammer et al, 1993) and reduced monoamine levels (Weiss et al, 1992; Parsons et al, 1995) in brain regions associated with reinforcement.

It has been suggested that somatic, affective, and neurochemical signs of cocaine withdrawal might enhance the reinforcing effects of cocaine by setting conditions under which cocaine could prevent or reverse these aversive abstinence signs and thereby function as a 'negative reinforcer' (Ahmed and Koob, 2005; Koob, 2009). Evidence to address this hypothesis is primarily derived from studies using progressive-ratio procedures. For example, increased breakpoints for cocaine were found after some periods of cocaine exposure and withdrawal in rats (Paterson and Markou, 2003; Morgan et al, 2002, 2005; Wee et al, 2008; Orio et al, 2009). However, multiple other regimens of cocaine exposure and withdrawal failed to increase breakpoints maintained by cocaine in rats or nonhuman primates, and in some cases, breakpoints decreased during cocaine withdrawal (Yanagita, 1975; Yanagita, 1980; Li et al, 1994; Morgan and Roberts, 2004; Liu et al, 2005; Czoty et al, 2006). Taken together, these studies provide much weaker evidence for withdrawal-associated increases in cocaine reinforcement than exists for withdrawal-associated increases in opioid reinforcement.

The aim of this study was to examine the effects of extended cocaine access and subsequent withdrawal on the relative reinforcing strength of cocaine using a novel cocaine $v s$ food choice procedure in monkeys. The choice procedure was used for two reasons. First, we and others have shown earlier that opioid withdrawal increases choice of heroin or morphine over food in opioid-dependent monkeys (Spragg, 1940; Griffiths et al, 1975; Negus, 2006; Negus and Rice, 2009). Consequently, effects of cocaine withdrawal could be examined under conditions similar to those used earlier to study withdrawal-associated increases in opioid reinforcement. Second, the primary-dependent variable in choice procedures (percent drug choice) is a measure of response allocation rather than response rate. As a result, this measure may be less sensitive than progressive-ratio breakpoints or other dependent measures to nonselective effects of drug withdrawal on overall response rates, which might mask withdrawal-induced increases in reinforcing strength (Griffiths et al, 1975; Negus, 2003). We hypothesized that exposure to or withdrawal from extended cocaine access would increase choice of cocaine $v s$ food.

\section{METHODS}

\section{Animals}

Studies were conducted in four adult male rhesus monkeys (Macaca mulatta) that had been surgically implanted with double-lumen catheters using aseptic procedures as described earlier (Negus, 2003). Monkeys weighed 6-10 kg and were maintained on a diet of multiple vitamins, fresh fruit, and food biscuits (Lab Diet Jumbo Monkey Biscuits, PMI Feeds, Inc, St Louis, MO). Biscuits and vitamins were provided in the morning at approximately 9 a.m., and fruit was provided daily between 4 and 5 p.m. In addition, monkeys received up to $50 \mathrm{1-g}$ banana-flavored pellets (Precision Primate Pellets Formula L/I Banana Flavor, P. J. Noyes Co, Lancaster, $\mathrm{NH}$ ) during daily operant sessions (see below). Water was continuously available. A 12-h light-dark cycle was in effect (lights on from 7 a.m. to 7 p.m.). All monkeys had prior exposure to the cocaine $v s$ food choice procedure and treatment with monoaminergic and/or opioid test compounds (unpublished results). However, this study was the first exposure for any monkey to the extended cocaine access conditions.

Animal maintenance and research were conducted in accordance with the Guide for the Care and Use of Laboratory Animals as adopted and promulgated by the National Institutes of Health. The facility was licensed by the United States Department of Agriculture, and the Institutional Animal Care and Use Committee approved all protocols. Furthermore, consulting veterinarians periodically monitored the health of the monkeys. Monkeys had visual, auditory, and olfactory contact with other monkeys throughout the study. Operant procedures and foraging toys provided opportunities for environmental manipulation and enrichment. Music or nature videotapes were also played daily in animal housing rooms to provide additional environmental enrichment.

\section{Apparatus and Catheter Maintenance}

Experimental sessions were conducted in each monkey's home cage. The front wall was equipped with an operant response panel $\left(28 \times 28 \mathrm{~cm}^{2}\right)$ that included three circular response keys $(5.1 \mathrm{~cm}$ in diameter) arranged $2.5 \mathrm{~cm}$ apart horizontally. Red, green, or yellow stimulus lights could illuminate each key. Each housing chamber was also equipped with a pellet dispenser (Gerbrands, Model G5210, Arlington, MA) and two syringe pumps (Model BSP-lE, Braintree Scientific, Braintree, MA; or Model PHM-100, Med Associates Inc, St Albans, VT), one for each lumen of the double-lumen catheter. One syringe pump (the 'selfadministration pump') was used to deliver self-administered cocaine injections through one lumen of the double-lumen catheter. The second syringe pump was used to deliver saline through the second lumen of the catheter to promote catheter patency. This second pump was programmed to deliver $0.1 \mathrm{ml}$ infusions every $20 \mathrm{~min}$ from 10 a.m. each day until 9 a.m. the next morning. From 9 a.m. to 10 a.m. each morning, the health of the monkeys was 
inspected, equipment and syringe volumes were checked, and syringes were replaced if necessary. Operation of the operant response panels and data collection were accomplished with microprocessors and software purchased from Med Associates Inc. The intravenous catheter was protected by a tether system consisting of a custom-fitted nylon vest connected to a flexible stainless steel cable and fluid swivel (Lomir Biomedical, Malone, NY). This flexible tether system permitted monkeys to move freely in the cage. Catheter patency was periodically evaluated by i.v. administration of ketamine $(5 \mathrm{mg} / \mathrm{kg})$ or the short-acting barbiturate methohexital $(3 \mathrm{mg} / \mathrm{kg})$ through the catheter lumen. The catheter was considered to be patent if i.v. administration of ketamine or methohexital produced a loss of muscle tone within $10 \mathrm{~s}$.

\section{Behavioral Procedures}

Training procedures. Behavioral sessions were conducted 7 days a week as described earlier (Negus, 2003). After initial shaping of key press responding maintained by food delivery (1-g food pellets) and drug injections $(0.1 \mathrm{mg} / \mathrm{kg} /$ injection cocaine), choice training was initiated. Choice sessions were conducted during daily 2-h sessions from 11 a.m. to 1 p.m. The terminal choice schedule consisted of five 20-min components separated by $5 \mathrm{~min}$ time-out periods (total session duration of $120 \mathrm{~min}$ ). During each component, the left, food-associated key was illuminated with red stimulus lights, and completion of the fixed-ratio (FR) requirement resulted in the delivery of a food pellet. The right, cocaine-associated key was illuminated with green stimulus lights, and completion of the FR requirement on this key resulted in the delivery of a cocaine dose. A different cocaine dose was available during each of the five successive components $(0,0.0032,0.01,0.032$, and $0.1 \mathrm{mg} / \mathrm{kg} /$ injection during components $1-5$, respectively), and dose was varied by varying the duration of pump activation and the resulting volume of each injection. Stimulus conditions on the drug-associated key were also varied by flashing the stimulus lights on and off in 3-s cycles. (Component 1: $0 \mathrm{~s}$ on, $3 \mathrm{~s}$ off; Component 2: $0.1 \mathrm{~s}$ on, $2.9 \mathrm{~s}$ off; Component 3: $0.3 \mathrm{~s}$ on, $2.7 \mathrm{~s}$ off; Component 4: $1 \mathrm{~s}$ on, $2 \mathrm{~s}$ off; Component 5: $3 \mathrm{~s}$ on, $0 \mathrm{~s}$ off). Thus, longer flashes (and shorter inter-flash intervals) were associated with higher available drug doses. The response requirements were set at FR 100 on the food-associated key and FR 10 on the cocaine-associated key for all monkeys, because our earlier studies indicated that, under these response requirements, monkeys usually switched from the food-associated key to the drug-associated key during the fourth response period, when an intermediate unit dose of $0.032 \mathrm{mg} / \mathrm{kg} /$ injection cocaine was available (Negus, 2003). An ascending dose order was used because we found earlier that dose order had little or no effect on the cocaine choice doseeffect curve (Negus, 2003). Consequently, with the procedures used in this study, it was possible to observe both leftward and rightward shifts in the cocaine choice doseeffect curves that might result from manipulation of experimental variables.

During each component, monkeys could complete up to 10 total ratio requirements on the food- and cocaineassociated keys. Responding on either key reset the ratio requirement on the other key. Completion of each ratio requirement initiated a 30-s time-out, during which all stimulus lights were turned off, and responding had no scheduled consequences. During components when the drug-associated key was not illuminated and a ' 0 ' dose of drug was available, responses on this key were still recorded, they still reset the FR requirement on the foodassociated key, and completion of the FR requirement still counted as one of the 10 allotted ratios and initiated a 30-s time-out. If all 10 ratio requirements were completed before the 20-min component had elapsed, then all stimulus lights were extinguished and responding had no scheduled consequences for the remainder of that 20-min component. Choice training was considered to be complete when the lowest cocaine dose maintaining at least $80 \%$ cocaine choice varied by $\leqslant 0.5 \log$ units for 3 consecutive days.

Testing procedures. Once responding under the choice schedule was stable, daily choice sessions were continued, and additional daily 'supplemental sessions' of cocaine availability were introduced as described below to provide extended access to cocaine self-administration. Procedures for introducing extended cocaine access were identical to procedures used earlier to study effects of dependence and withdrawal associated with extended heroin access on heroin vs food choice (Negus, 2006; Negus and Rice, 2009). In this study, daily supplemental sessions began at 1 p.m. (ie, immediately after the choice session) and concluded the next morning at 10 a.m. During this 21-h supplemental session, the cocaine-associated key was illuminated with green lights, and cocaine $(0.1 \mathrm{mg} / \mathrm{kg} /$ injection) was available under a FR 10/time-out $\mathrm{X}$ min schedule. Three time-out values $(30,15$, and $7.5 \mathrm{~min})$ were investigated to provide graded levels of extended cocaine access. A 30-min time-out permitted a maximum of 42 injections $(4.2 \mathrm{mg} / \mathrm{kg})$ during the 21 -h supplemental session, whereas a 15 -min time-out permitted a maximum of 84 injections $(8.4 \mathrm{mg} / \mathrm{kg})$, and a $7.5-\mathrm{min}$ time-out permitted a maximum of 168 injections $(16.8 \mathrm{mg} / \mathrm{kg})$.

Supplemental sessions were studied in a descending order of time-out values $(30,15$, and $7.5 \mathrm{~min})$. Sessions using a given time-out period were implemented for a period of 7 consecutive days. At the conclusion of each 7-day period of extended access, supplemental sessions were terminated for a period of at least 7 days and until responding during the choice sessions recovered to baseline levels for at least 3 days. Supplemental sessions with the next time-out value were then implemented for 7 days, and so on. In two cases, exposure to supplemental sessions with the shortest timeout duration $(7.5 \mathrm{~min})$ was extended to 8 days, because a sharp but transient reduction in intake occurred on day 7 (a 'crash,' see below).

A goal of this study was to determine whether extended cocaine access or cocaine withdrawal would alter cocaine $v s$ food choice in a manner comparable to the way in which heroin withdrawal was shown to alter heroin $v s$ food choice (Negus, 2006; Negus and Rice, 2009). However, earlier studies have suggested that withdrawal-associated changes in cocaine self-administration may be delayed and may occur only after a period of complete abstinence from cocaine (Morgan et al, 2002). Accordingly, a follow-up 
study was also conducted. In this follow-up study, supplemental sessions with a 7.5-min time-out were implemented for 7 days (from days 1-7). At the conclusion of this 7-day period, supplemental sessions were terminated. In addition, cocaine access during daily choice sessions was also withheld for a second period of 7 days (from days 8 to 14). During this time, food continued to be available during all five components of the daily choice sessions, but the cocaine key was not illuminated, and completion of the response requirement on the cocaine key did produce a cocaine injection. On day 15, normal cocaine access was reinstated during daily choice sessions (ie, unit doses of 0 , $0.0032,0.01,0.032$, and $0.1 \mathrm{mg} / \mathrm{kg} /$ injection cocaine were available during the five sequential components of daily choice sessions). Behavior was then monitored for an additional 7 days (from days 15 to 21 ).

Assessment of somatic withdrawal signs. Approximately $1 \mathrm{~h}$ before each choice session during periods of cocaine withdrawal, the presence of somatic withdrawal signs was evaluated using a scoring system used earlier to score signs of opioid withdrawal (Negus, 2006; Negus and Rice, 2009). Specifically, eight withdrawal signs were counted as present or absent during each withdrawal assessment, and the total number of withdrawal signs were counted to yield a Withdrawal Score (ie, maximum Withdrawal Score was ' 8 '). The eight signs were lying on bottom of cage, unusually aggressive or lethargic response to investigator, increased vocalization, retching/emesis, diarrhea, penile erection/ masturbation, tremor/convulsion, and a category for other unusual behaviors.

\section{Data Analysis}

Choice sessions. The primary-dependent variables for each component were (1) percent cocaine choice, defined as (number ratios completed on the cocaine-associated key$\div$ number of ratios completed) $\times 100$ and (2) the number of ratios completed. These variables were then plotted as a function of cocaine dose. The ED50 value of the cocaine choice dose-effect curve was defined as the dose of cocaine that produced $50 \%$ cocaine choice. ED50 values were calculated by interpolation when only two data points were available (one below and one above $50 \%$ cocaine choice) or by linear regression when at least three data points were available on the linear portion of the dose-effect curve. Log ED50 values were calculated for each monkey during (1) the last 3 'baseline' days before introduction of each type of supplemental session, (2) the last 3 days of each 7-day period of access to supplemental sessions, and (3) days 1, 4, and 7 after exposure to each type of supplemental session (or days 1, 4, and 7 after the 7-day abstinence period for the follow-up study). Additional dependent variables collected during each session included total choices, total food choices, total cocaine choices, and total cocaine intake.

The initial phase of the study, which examined the effects of extended cocaine access provided through supplemental sessions with different time-out values, was conducted in a group of four monkeys. Baseline data collected before implementation of each type of supplemental session were similar, and these data were averaged for display and analysis. The second phase of the study, which examined the effects of 7 days extended access followed by 7 days of cocaine abstinence, was conducted in a group of three monkeys, and baseline data collected only during this phase of the study were used for display and analysis. Values were compared by one-factor ANOVA, with treatment condition as a within-subjects factor. A significant ANOVA was followed by the Dunnett post hoc test to compare test conditions with baseline conditions. The criterion for significance was set at $P<0.05$.

Supplemental sessions. The primary-dependent variables from supplemental sessions were the number of injections per day and the amount of cocaine intake per day in $\mathrm{mg} / \mathrm{kg}$. In addition, to provide information on diurnal patterns of cocaine self-administration during supplemental sessions, the number of injections during each quintile of each supplemental session was also determined. The timing of the five quintiles was 1:00-5:12 p.m., 5:12-9:24 p.m., 9:24 p.m.-1:36 a.m., 1:36-5:48 a.m., and 5:48-10:00 a.m.

\section{Drugs}

Cocaine $\mathrm{HCl}$ (provided by the National Institute on Drug Abuse Drug Supply Program, Bethesda, MD) was dissolved in sterile saline. Drug doses are expressed in terms of this salt form of cocaine.

\section{RESULTS}

\section{Baseline Choice Between Cocaine and Food}

Figure 1 (top panel, open circles) shows that cocaine maintained a dose-dependent increase in cocaine choice under baseline conditions, when cocaine was available only during daily choice sessions. When low cocaine doses were available $(0-0.01 \mathrm{mg} / \mathrm{kg} / \mathrm{injection})$, monkeys responded almost exclusively for food, and when higher cocaine doses were available (0.032-0.1 mg/kg/injection), monkeys responded almost exclusively for cocaine. Monkeys usually earned the maximum of 10 reinforcers during each component of the choice session (Figure 1, bottom panel, open circles).

The baseline cocaine choice ED50 value is shown in Table 1. The value shown in Table 1 is the mean of three baseline determinations conducted before the introduction of supplemental sessions with each of the three time-out durations. These baseline data were averaged for display and analysis because baseline dose-effect were stable across determinations. Specifically, baseline choice ED50 values were $0.021 \mathrm{mg} / \mathrm{kg}(0.011-0.039)$ before 30 -min time-out supplemental sessions, $0.018 \mathrm{mg} / \mathrm{kg}(0.011-0.031)$ before 15 -min time-out supplemental sessions, and $0.019 \mathrm{mg} / \mathrm{kg}$ (0.014-0.025) before 7.5-min time-out supplemental sessions.

\section{Effects of Extended Cocaine Access on Choice Between Cocaine and Food}

Figure 1 and Table 1 also show cocaine choice dose-effect curves and ED50 values during periods of extended cocaine access produced by introducing daily, 21-h supplemental sessions of cocaine self-administration with 30,15 , or 


\section{-O- Baseline \\ $\longrightarrow+$ +Supplemental Session (30'TO) \\ - +Supplemental Session (15'TO) \\ +Supplemental Session (7.5'TO)}
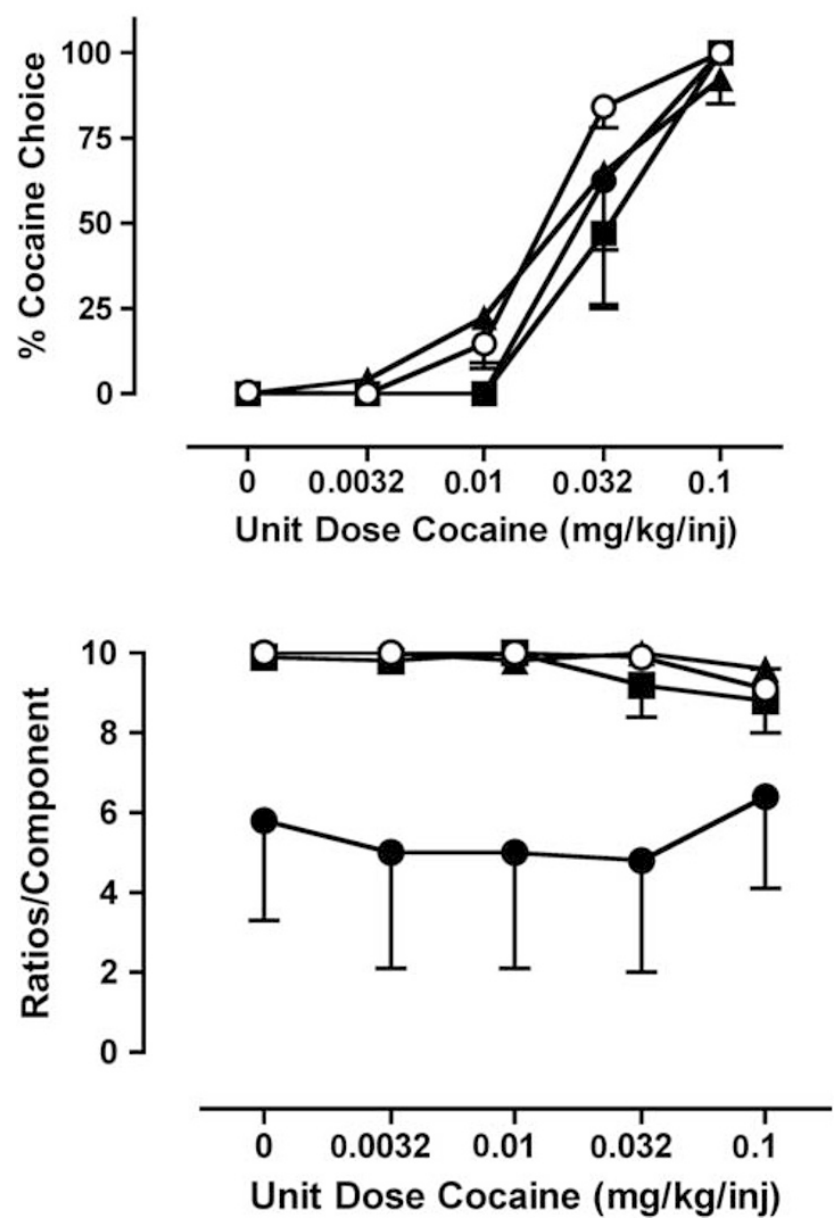

Figure I Effects of extended cocaine access on cocaine choice in rhesus monkeys. Abscissae: unit dose cocaine ( $\mathrm{mg} / \mathrm{kg} /$ injection). Ordinate (top panel): \% Cocaine choice. Ordinate (bottom panel): number of ratios completed per component (maximum $=10$ ratios per component). Extended cocaine access was accomplished by introducing daily, 21-h supplemental sessions of cocaine availability using 30,15 , and 7.5 min postinjection time-out periods. Baseline data show mean results from the 3 days preceding each 7-day period of extended access. Supplemental session data show mean results from the last 3 days of each 7-day period of extended access. All points show mean \pm SEM from four monkeys except the filled circles in the top panel (\% cocaine choice during exposure to supplemental sessions with 7.5 min post-injection time-outs). These points show data for only 2-3 monkeys, because two monkeys failed to respond during most components and \% cocaine choice could not be calculated.

7.5 min time-out periods after each injection. There was a trend for extended cocaine access to produce small rightward shifts in mean cocaine choice dose-effect curves, but extended cocaine access did not significantly alter cocaine choice ED50 values. Extended cocaine access also reduced the numbers of reinforcers/component. During exposure to supplemental sessions with the shortest post-injection time-out $(7.5 \mathrm{~min})$, two of the four monkeys failed to
Table I Mean Cocaine ED50 Values in mg/kg (95\% CL) Under Baseline Conditions, During 7-day Access to Supplemental Cocaine Self-Administration Sessions with 30, 15, and 7.5 min Post-Injection Time-Outs, and on Days I, 4, and 7 After Termination of Access to Supplemental Sessions

\begin{tabular}{lc}
\hline Condition & Cocaine ED50 in $\mathbf{~ m g / k g}(\mathbf{9 5} \% \mathbf{C L})$ \\
\hline Baseline & $0.019(0.014-0.027)$ \\
Supplemental sessions (30' TO) & \\
$\quad$ During access & $0.021(0.008-0.058)$ \\
Post day I & $0.021(0.011-0.042)$ \\
Post day 4 & $0.013(0.004-0.038)$ \\
Post day 7 & $0.013(0.004-0.039)$ \\
& \\
Supplemental sessions ( $15^{\prime}$ TO) & \\
During access & $0.038(0.023-0.062)$ \\
Post day I & $0.031(0.017-0.059)$ \\
Post day 4 & $0.021(0.015-0.029)$ \\
Post day 7 & $0.028(0.016-0.047)$ \\
Supplemental sessions (7.5' TO) & \\
During access & Not determined ${ }^{\mathrm{a}}$ \\
Post day I & Not determined \\
Post day 4 & $0.018(0.007-0.045)$ \\
Post day 7 & $0.018(0.018-0.018)$ \\
\hline Cocane ED &
\end{tabular}

Cocaine ED50 values were not significantly different from baseline either during or after extended cocaine access.

${ }^{a}$ Not determined because rate-decreasing effects prevented calculation of ED50 values in some monkeys.

respond for either food or cocaine during most components of most daily choice sessions, and responding was also disrupted to a lesser degree in a third monkey.

\section{Patterns of Cocaine Self-Administration During Extended Cocaine Access}

Although extended cocaine access had little effect on cocaine choice dose-effect curves, the conditions of extended access did produce dramatic increases in daily cocaine intake. Figure 2 shows the number of injections delivered each day in each monkey during supplemental sessions with postinjection time-outs of 30,15 , or $7.5 \mathrm{~min}$. In general, reductions in the post-injection time-out periods increased the numbers of injections/day. During supplemental sessions with the shortest time-outs $(7.5 \mathrm{~min})$, monkeys often responded for $>100$ injections/day. Reductions in post-injection time-outs also increased the day-to-day variability in cocaine self-administration. This effect was especially prominent in monkeys 21552 and 21786 during supplemental sessions with the shortest post-injection timeout period $(7.5 \mathrm{~min})$. For both monkeys, days with high rates of cocaine self-administration alternated with days of little or no self-administration. This type of pattern has been referred to earlier as an 'erratic' or a 'binge-crash' pattern of cocaine self-administration (Deneau et al, 1969; Siegel, 1982; Markou and Koob, 1991). 


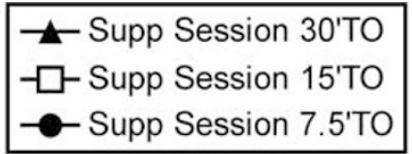

$\operatorname{cox}$

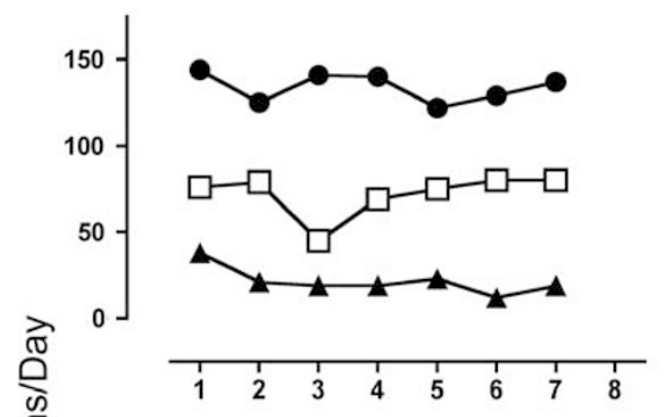

21552

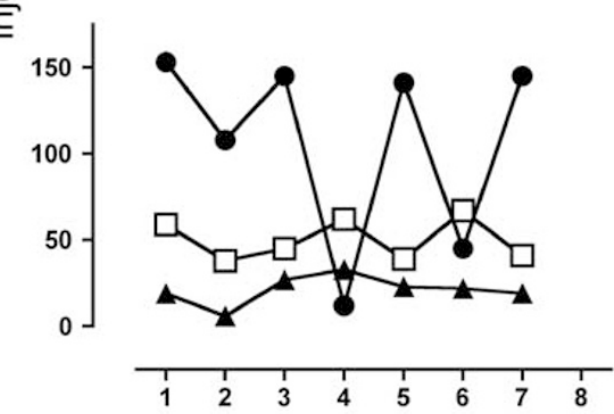

RQ2393
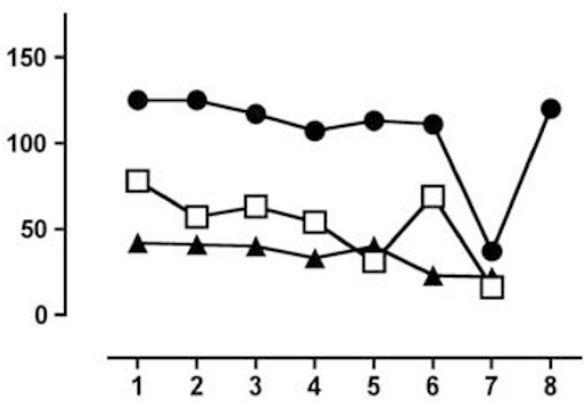

21786

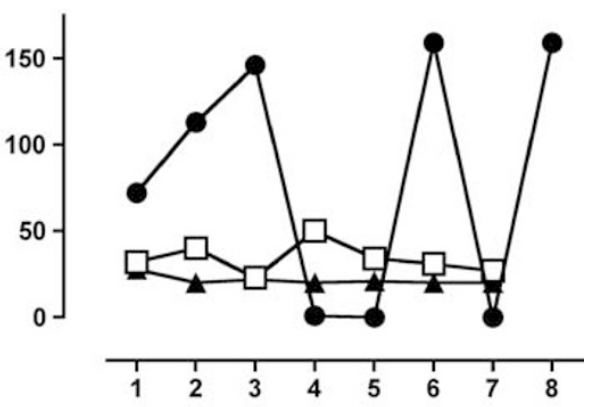

Consecutive Days of Access

Figure 2 Cocaine self-administration by individual rhesus monkeys during extended cocaine access (supplemental sessions of cocaine availability with post-injection time-outs of 30, I5, or $7.5 \mathrm{~min}$ ). Abscissae: consecutive days of access. Ordinates: number of cocaine injections (0.I mg/kg/injection) earned per experimental day. The identification number of each monkey is shown at the top of the panel. Each point shows data from a single determination in one monkey.

Figure 3 (left panel) shows the diurnal pattern of cocaine self-administration across each of the five quintiles of the 21-h supplemental sessions. Reductions in the post-injection time-out period increased rates of cocaine selfadministration during each quintile of the supplemental sessions. Diurnal patterns of self-administration were also disrupted. During supplemental sessions with the longest post-injection time-outs $(30 \mathrm{~min})$, monkeys selfadministered cocaine primarily during the afternoon/ evening (quintiles 1 and 2 from 1:00-9:24 p.m.) and during the morning (quintile 5 from 5:48-10 a.m.). Monkeys selfadministered very little cocaine at night (quintiles 3 and 4 from 9:24 p.m.-5:48 a.m.). Conversely, during supplemental sessions with the shortest post-injection time-outs (7.5 min), monkeys self-administered cocaine throughout all five quintiles, with a tendency for self-administration to decline across sequential quintiles.

Figure 3 (right panel) shows daily cocaine intake under baseline conditions (choice sessions only) and during extended cocaine access (choice sessions + supplemental sessions). Under baseline conditions, cocaine intake was $1.19 \mathrm{mg} / \mathrm{kg} /$ day. Cocaine intake increased with the introduction of supplemental sessions. During access to supplemental sessions with the shortest post-injection time-outs
(7.5 min), mean cocaine intake increased approximately 10 -fold over baseline levels to $11.57 \mathrm{mg} / \mathrm{kg}$.

\section{Effects of Withdrawal from Extended Cocaine} Access on Choice Between Cocaine and Food

Figure 4 shows cocaine choice dose-effect curves determined 1, 4, and 7 days after termination of extended access to cocaine. Cocaine choice ED50 values are shown in Table 1. In general, withdrawal from extended cocaine access had little effect on cocaine choice at any of the time points. In addition, withdrawal from extended cocaine excess failed to elicit any somatic withdrawal signs at any time in any monkey (data not shown). However, there was a time-dependent recovery in the numbers of reinforcers/component after termination of supplemental sessions with $7.5 \mathrm{~min}$ post-injection time-outs (Figure 4, bottom right panel).

\section{Effects of Exposure to and Withdrawal from Extended Cocaine Access on Total Choices, Food Choices, Cocaine Choices, and Cocaine Intake}

Figure 5 shows the number of total choices, food choices, and cocaine choices, and the level of cocaine intake during 
choice sessions conducted under baseline conditions, during extended access to cocaine, and on days 1, 4, and 7 after termination of extended access. Exposure to and withdrawal from supplemental sessions with 30 and $15 \mathrm{~min}$ time-outs had little effect on these endpoints. Exposure to supplemental sessions with $7.5 \mathrm{~min}$ time-outs reduced total choices, food choices, cocaine choices, and cocaine intake in most monkeys, but this effect did not achieve statistical significance for the group. There was also a tendency for
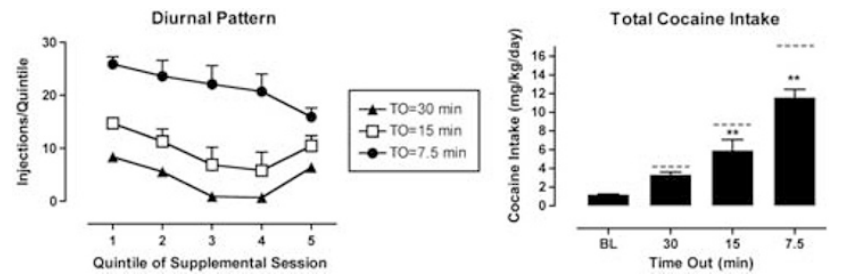

Figure 3 Cocaine intake during periods of extended cocaine access. Left panel: diurnal patterns of cocaine during supplemental sessions of cocaine availability with post-injection time-outs of 30,15 , or $7.5 \mathrm{~min}$. Abscissa: Quintiles of the 21-h supplemental session (Quintile 1: 1:00-5:12 p.m.; Quintile 2: 5:12-9:24 p.m.; Quintile 3: 9:24 p.m.-I:36 a.m.; Quintile 4: I:365:48 a.m.; Quintile 5: 5:48-10:00 a.m.). Ordinate: number of cocaine injections (0.1 mg/kg/injection) during each quintile. Each point shows mean \pm SEM from four monkeys during the last 3 days of exposure to each type of supplemental session. Right panel: daily cocaine intake at baseline and during access to supplemental sessions. Abscissa: duration of the postinjection time-out period during the supplemental sessions. The bar above 'BL' shows baseline data when there were no supplemental sessions and cocaine was available only during choice sessions. Ordinate: daily cocaine intake in $\mathrm{mg} / \mathrm{kg}$. Baseline data show results from the 3 days preceding each 7-day period of extended access. Supplemental session data show results from the last 3 days of each 7-day period of extended access. All bars show mean \pm SEM in four monkeys. Asterisks indicate a significant difference from baseline. ${ }^{* *} p<0.01$. Dashed bar above each supplemental session indicates total possible cocaine intake. each of these parameters to recover during the 7 days after termination of supplemental sessions.

\section{Effects of Withdrawal from Extended Cocaine Access + 7-day Abstinence on Choice Between Cocaine and Food}

Figure 6 shows cocaine choice dose-effect curves on days 1, 4 , and 7 after (1) a 7-day period of extended access to cocaine (supplemental sessions with $7.5 \mathrm{~min}$ post-injection time-outs), followed by (2) a 7-day period of total cocaine abstinence (no cocaine available during either choice or supplemental sessions). Cocaine choice ED50 values are shown in Table 2. As in the initial phase of the study, cocaine maintained a dose-dependent increase in cocaine choice under baseline conditions, and extended cocaine access increased daily cocaine intake (from $1.31 \pm 0.01$ to $10.11 \pm 0.96 \mathrm{mg} / \mathrm{kg} /$ day) while having little effect on the cocaine choice dose-effect curve and decreasing the number of ratios completed per component. After termination of extended access, monkeys were exposed to 7 days of cocaine abstinence when only food was available during daily choice sessions, and all monkeys responded for all 50 available food pellets each day during this period of cocaine abstinence. When cocaine choice was reinstated after this period of extended cocaine access and cocaine abstinence, cocaine choice dose-effect curves were similar to baseline dose-effect curves, and cocaine ED50 values were not different from baseline. Total choices, food choices, cocaine choices, and cocaine intake were also similar to baseline levels (data not shown).

\section{DISCUSSION}

This study examined effects of exposure to and withdrawal from extended cocaine access on the relative reinforcing
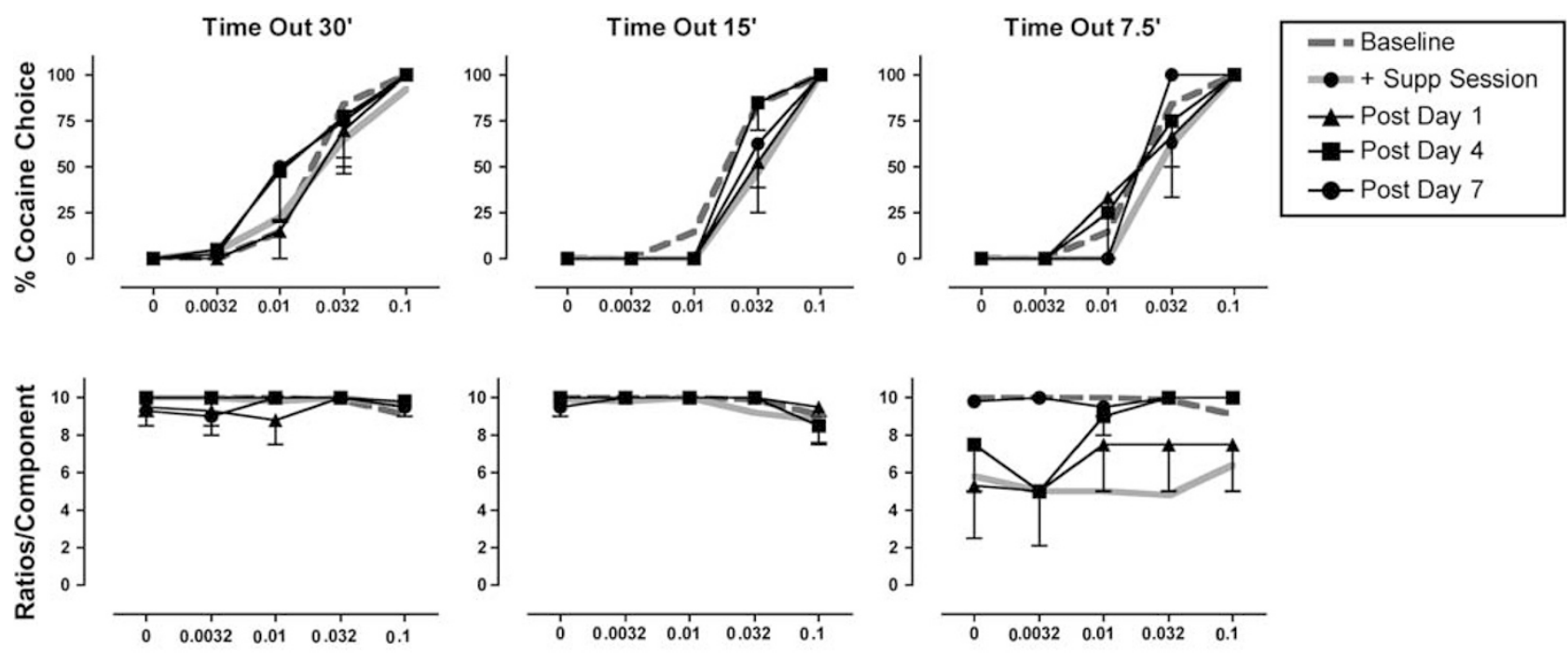

Unit Dose Cocaine (mg/kg/inj)

Figure 4 Effects of withdrawal from extended cocaine access on cocaine choice. Abscissae: unit dose cocaine in mg/kg/injection. Ordinates (top panels): percent cocaine choice. Ordinates (bottom panels). Number of ratios completed per component. Data are shown in the left, center, and right panels for supplemental sessions with 30, 15, and 7.5 min post-injection time-outs, respectively. In each panel, baseline data are indicated by a dotted gray line, and data obtained during access to supplemental sessions are shown by a solid gray line (identical to data shown in Figure I). All points show mean \pm SEM data from four monkeys collected on days I, 4, and 7 after termination of access to supplemental sessions. 

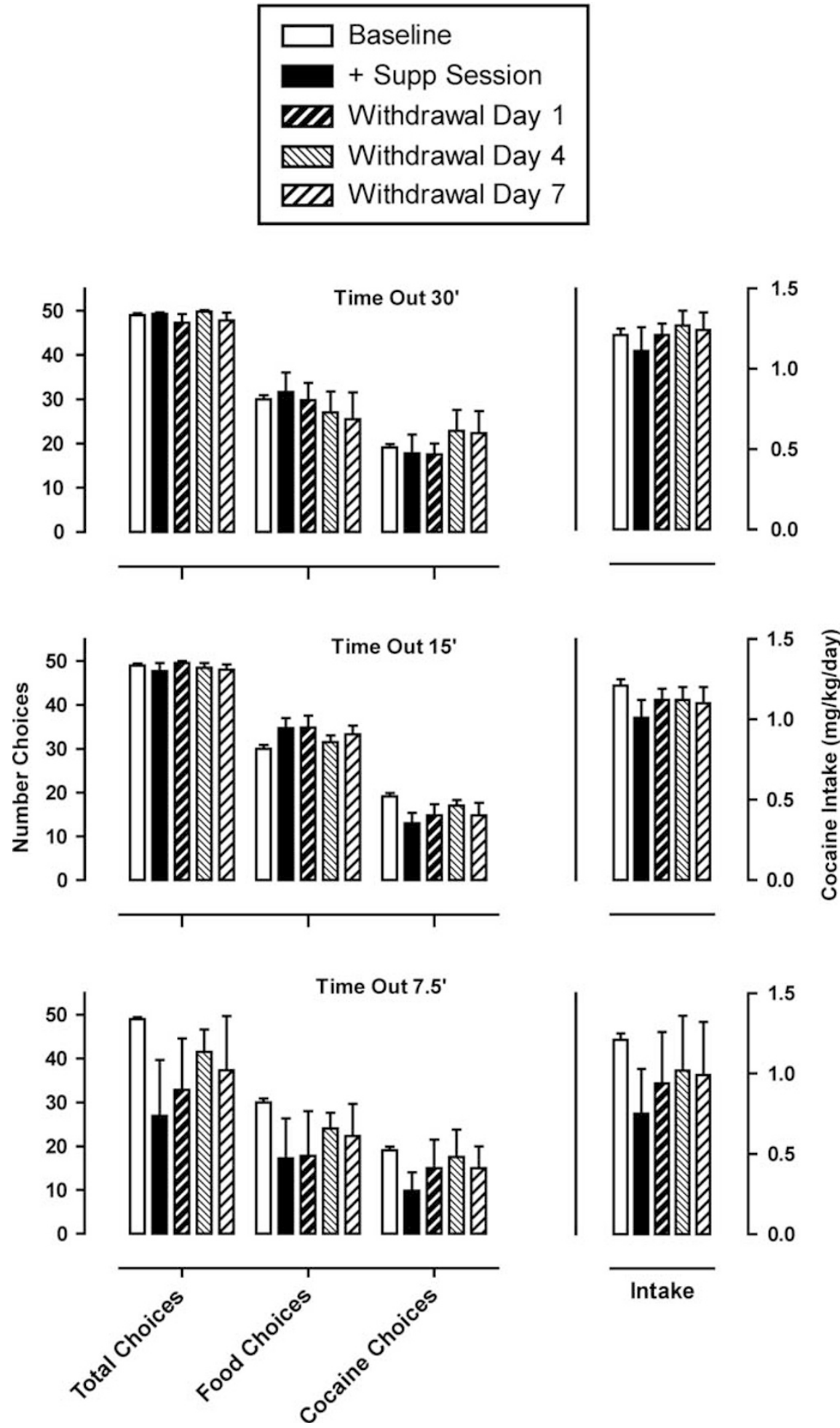

Figure 5 Total choices, food choices, cocaine choices, and cocaine intake during choice sessions before, during, and after extended cocaine access. Abscissae: experimental endpoint. Left ordinates: number of choices per session. Right ordinates: cocaine intake in mg/kg/day during choice sessions. All bars show mean \pm SEM data from four monkeys. None of the parameters were significantly different from baseline either during or after extended cocaine access.

strength of cocaine in a cocaine $v s$ food choice procedure in rhesus monkeys. Although conditions of extended access were sufficient to maintain high daily cocaine intakes and produce 'binge-crash' patterns of cocaine self- administration, withdrawal did not increase choice of cocaine over food. Consequently, these results do not support the hypothesis that cocaine withdrawal increases the reinforcing strength of cocaine. The relatively modest 

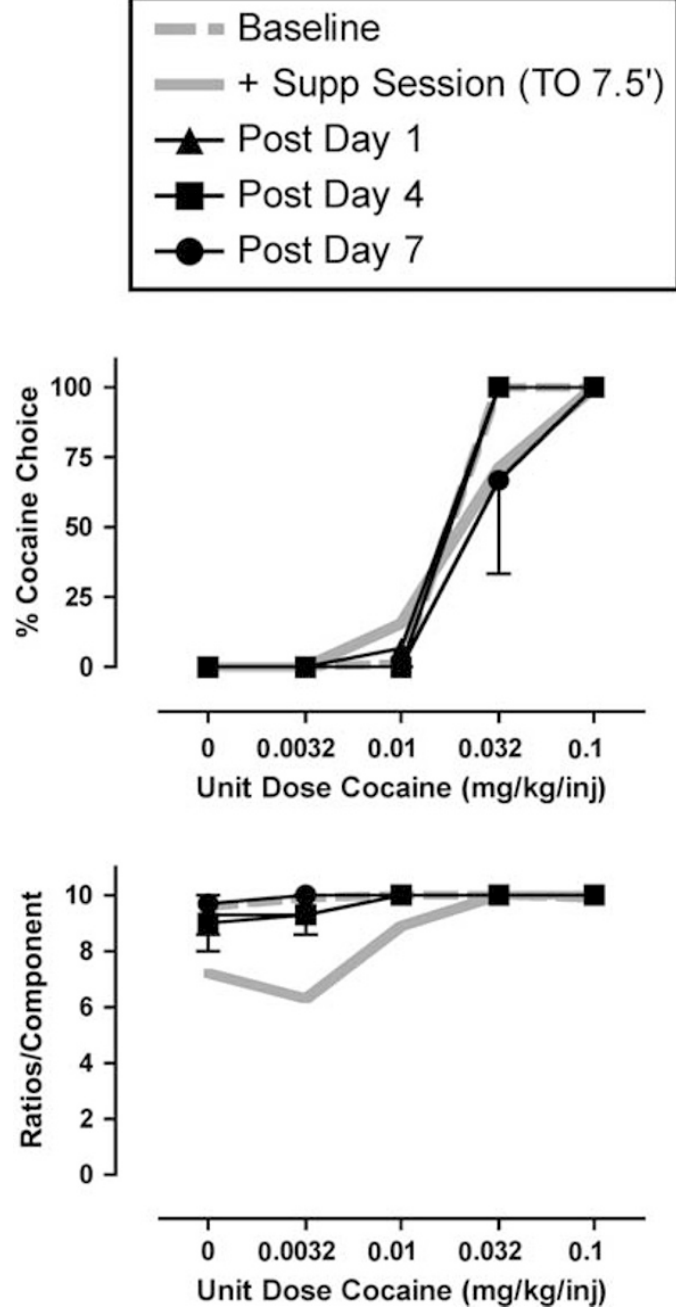

Figure 6 Effects of extended cocaine access +7 days of cocaine abstinence on cocaine choice. Abscissae: unit dose cocaine in $\mathrm{mg} / \mathrm{kg} /$ injection. Ordinate (top panel): percent cocaine choice. Ordinate (bottom panel). Number of ratios completed per component. In each panel, baseline data are indicated by a dotted gray line, and data obtained during access to supplemental sessions ( $7.5 \mathrm{~min}$ post-injection time-outs) are shown by a solid gray line. All points show mean \pm SEM from three monkeys collected on days 1, 4, and 7 after the 7-day abstinence period (ie, days $8,1 \mathrm{I}$, and 14 after termination of access to supplemental cocaine).

effects of cocaine withdrawal on cocaine reinforcement may have implications for both the mechanisms and treatment of cocaine addiction.

\section{Cocaine Choice Before and During Extended Cocaine Access}

This study evaluated the relative reinforcing strength of cocaine using a concurrent-choice schedule of cocaine and food availability. Under baseline conditions before introduction of extended cocaine access, cocaine maintained a dose-dependent increase in cocaine choice. These results are consistent with earlier studies of cocaine $v s$ food choice in rhesus monkeys (Nader and Woolverton, 1992; Paronis et al, 2002; Negus, 2003). Conditions of extended drug access produced graded increases in daily cocaine intakes that were sufficient to decrease the total number of ratios
Table 2 Mean Cocaine ED50 Values in $\mathrm{mg} / \mathrm{kg}$ (95\% CL) Under Baseline Conditions, During 7-day Access to Supplemental Cocaine Self-Administration Sessions 7.5 min Post-Injection Time-Outs, and on Days I, 4, and 7 After Termination of Access to Supplemental Sessions +7 Days of Cocaine Abstinence

\begin{tabular}{lc}
\hline Condition & Cocaine ED50 in $\mathbf{~ m g / k g ~ ( 9 5 \% ~ C L ) ~}$ \\
\hline Baseline & $0.018(0.018-0.018)$ \\
During access & $0.023(0.011-0.047)$ \\
Post abstinence day I & $0.017(0.015-0.019)$ \\
Post abstinence day 4 & $0.018(0.018-0.018)$ \\
Post abstinence day 7 & $0.026(0.013-0.055)$
\end{tabular}

Cocaine ED50 values were not significantly different from baseline either during extended cocaine access or after the 7-day abstinence period.

completed during choice sessions. However, there was little change in cocaine choice dose-effect curves during extended cocaine access. These effects produced by contingent cocaine agree with the finding that noncontingent cocaine decreased cocaine self-administration under choice or multiple schedules only at doses that also decreased food-maintained responding (Glowa and Fantegrossi, 1997; Panlilio et al, 1998; Negus, 2003).

\section{Effects of Cocaine Withdrawal on Cocaine Self-Administration}

Drug withdrawal in drug-dependent organisms has long been postulated to promote use of some addictive drugs. However, this study found that withdrawal from extended cocaine access failed to alter the reinforcing strength of cocaine as measured by cocaine $v s$ food choice. This finding suggests that drug withdrawal may have a lesser role in regulating the reinforcing strength of cocaine than of other drugs of abuse, such as opioids. Before addressing the implications of this possibility, three other possible explanations for our negative findings should also be considered.

First, one possibility is that drug $v s$ food choice is a relatively insensitive measure of reinforcing strength, and that this measure failed to detect changes in the reinforcing strength of cocaine produced by cocaine withdrawal. Two findings argue against this possibility. First, cocaine $v s$ food choice in this procedure is sensitive to many other manipulations that are thought to influence the relative reinforcing strength of cocaine, including magnitude of the drug and food reinforcers, FR values for the drug and food choices, noncontingent food delivery, punishment of food or drug choice, treatment with monoamine releasers, and social rank (Negus, 2003, 2004, 2005a, 2005b; Czoty et al, 2005). Second, opioid withdrawal in opioid-dependent rhesus monkeys produces a robust increase in choice of opioid agonists over food (Spragg, 1940; Griffiths et al, 1975; Negus, 2006; Negus and Rice, 2009). Thus, choice procedures in general are sensitive to many manipulations, and opioid choice is sensitive to opioid withdrawal.

Second, the choice measure in this study provides a relative measure of the reinforcing strength of cocaine in comparison to that of the alternative food reinforcer. As a 
result, it is possible that increases in cocaine's reinforcing strength were masked by a concurrent increase in the reinforcing strength of food to produce no net change in choice. Again, two sets of evidence argue against this possibility. First, cocaine withdrawal has been reported to produce either no effect or a decrease in response rates maintained by food (Carroll and Lac, 1987; Woolverton and Kleven, 1988; Branch and Sizemore, 1988; Clark and Poling, 1990) and by other positive reinforcers, such as electrical brain stimulation (Markou and Koob, 1991; Kenny et al, 2003). In addition, cocaine withdrawal did not produce significant signs of hyperphagia, hypophagia, or carbohydrate craving in cocaine-dependent humans (Kampman et al, 1998). We are aware of no evidence independent of this study to suggest that cocaine withdrawal could increase the reinforcing strength of food and mask increases in the reinforcing strength of cocaine. Second, the results of this study using a choice procedure agree with the preponderance of data from studies that have used progressive-ratio procedures to assess withdrawal-induced changes in the reinforcing strength of cocaine. Thus, in both rats (Li et al, 1994; Morgan et al, 2002; Morgan, 2004, 2005; Liu et al, 2005) and nonhuman primates (Yanagita, 1978, 1980; Czoty et al, 2006), withdrawal from chronic exposure to either contingent or noncontingent cocaine usually produced either no change or a decrease in breakpoints maintained by cocaine. For example, Czoty et al (2006) examined effects of withdrawal from several different extended cocaine access conditions that resulted in a range of cocaine intakes from 1.2 to $6.9 \mathrm{mg} / \mathrm{kg} / \mathrm{day}$, and none of these conditions increased breakpoints maintained by cocaine. Cocaine withdrawal has been reported to increase cocaine-maintained breakpoints in rats under some conditions (Morgan et al, 2002, 2005; Wee et al, 2008; Orio et al, 2009), but these findings have not been representative of the general literature, and the precise determinants of these findings have not been clearly established. Overall, the present results using a choice procedure are consistent with most results from studies using progressive-ratio procedures in finding that cocaine withdrawal usually fails to increase the reinforcing strength of cocaine.

Finally, a third alternative explanation could be that the regimen of cocaine exposure and/or withdrawal was not sufficient to increase cocaine's reinforcing strength. Earlier studies that examined effects of cocaine withdrawal in rhesus monkeys may lend support to this possibility (Woolverton and Kleven, 1988; Kleven and Woolverton, 1991). In these studies, withdrawal from continuous infusion with cocaine disrupted rates of food-maintained responding. However, this abstinence sign was apparent only after treatment with a high dose of $32 \mathrm{mg} / \mathrm{kg} / \mathrm{day}$ cocaine for 10-35 days. Treatment with lower cocaine doses for shorter treatment times was not sufficient to produce withdrawal-induced decreases in food-maintained responding. In this study, peak intakes of cocaine reached $11.57 \mathrm{mg} /$ $\mathrm{kg} / \mathrm{day}$, and these access conditions were implemented for only 7 days at a time. These access conditions were sufficient to engender higher daily cocaine intakes than those tested earlier in studies designed to assess effects of cocaine withdrawal on cocaine self-administration in rhesus monkeys (Czoty et al, 2006). Moreover, the cocaine access conditions in this study were sufficient to produce 'binge- crash' patterns of self-administration in $50 \%$ of the subjects and reductions in responding maintained by delivery of both cocaine and food during choice sessions. Conditions of greater access (eg, with shorter time-outs) were not tested out of concern for potential convulsive and lethal effects associated with unlimited cocaine access (Deneau et al, 1969; Aigner and Balster, 1978). Thus, this study assessed effects of withdrawal from functionally high levels of cocaine self-administration; however, careful studies to assess consequences of higher daily intakes for longer periods may be warranted.

\section{Implications for Mechanisms and Treatment of Cocaine Abuse}

The modest effects of cocaine withdrawal on cocaine's reinforcing strength may have implications for both the mechanisms and treatment of cocaine addiction. With regard to mechanism, there is a growing literature to describe both the behavioral and neurobiological consequences of chronic cocaine exposure and withdrawal (Koob, 2009). Moreover, it has been proposed that at least some of these consequences may contribute to addiction by contributing to conditions under which cocaine might serve as a negative reinforcer (Ahmed and Koob, 2005). However, results of this study and other related studies suggest that withdrawal from chronic cocaine may have relatively little effect on cocaine's reinforcing strength. Thus, although consequences of chronic cocaine exposure and withdrawal may be experimentally demonstrable and clinically relevant for overall health, they may have little to do with the maintenance of cocaine use.

With regard to medications development, drug withdrawal in cases of opioid dependence can increase the reinforcing effects not only of the abused opioid agonist (eg, heroin), but also of other opioid agonists that can be used as agonist treatment medications (eg, methadone). The withdrawal-associated enhancement in the reinforcing efficacy of opioid agonist medications may contribute to the relatively high rates of compliance with these medications and high rates of retention in many opioid agonist treatment programs (Kreek et al, 2002). By comparison, if cocaine withdrawal produces little change in the reinforcing efficacy of cocaine, then it may also produce little change in the reinforcing efficacy of candidate agonist medications. As a result, agonist-based approaches to the treatment of cocaine addiction may be deprived of a factor that contributes to the success of agonist-based treatments for opioid addiction. This finding may be partly responsible for the relatively low rates of retention in some recent clinical trials with otherwise effective agonist-based medications for cocaine dependence (Grabowski et al, 2001, 2004; Mooney et al, 2009). However, retention in opioid agonist treatment programs is also variable and influenced not only by the impact of withdrawal on the reinforcing strength of agonist medications, but also by a multitude of other factors related both to medication pharmacology (eg, adequate dosing) and to the environmental setting in which the medication is delivered (eg, contingency management) (Lowinson et al, 1997; Rhoades et al, 1998; Stitzer and Petry, 2006). Similarly, retention in agonist-based treatment programs for cocaine dependence will also be influenced 
by multiple factors, many of which remain to be optimized. Although cocaine withdrawal may have little impact on the reinforcing strength of agonist medications for cocaine dependence, the degree to which this single factor might limit the clinical effectiveness of these medications remains to be determined.

\section{ACKNOWLEDGEMENTS}

We thank Sam McWilliams and Ashley Bear for outstanding technical support and Kevin Costa for writing the computer programs used in this study. This work was funded exclusively from NIH grants RO1-DA026946, PO1DA14528, and T32-DA007027 from the National Institute on Drug Abuse, National Institutes of Health.

\section{DISCLOSURE}

Negus declares that during the past 3 years he has received compensation as a consultant for or collaborator with the pharmaceutical companies Alkermes, Argolyn, Grunenthal, and Limerick Biopharma for projects related to opioid pharmacology or assessment of abuse liability. This study was not related to any of these professional relationships. Banks declares that except for income received from his primary employer, no financial support or compensation has been received from any individual or corporate entity during the past 3 years for research or professional service and there are no personal financial holdings that could be perceived as constituting a potential conflict of interest.

\section{REFERENCES}

Ahmed SH, Koob GF (2005). Transition to drug addiction: a negative reinforcement model based on an allostatic decrease in reward function. Psychopharmacology 180: 473-490.

Aigner TG, Balster RL (1978). Choice behavior in rhesus monkeys: cocaine $v$ s food. Science 201: 534-535.

Basso AM, Spina M, Rivier J, Vale W, Koob GF (1999). Corticotropin-releasing factor antagonist attenuates the 'anxiogenic-like' effect in the defensive burying paradigm but not in the elevated plus-maze following chronic cocaine in rats. Psychopharmacology 145: 21-30.

Branch MN, Sizemore GM (1988). Behavioral tolerance to cocaine in squirrel monkeys: acute and chronic effects on complex operant behavior. Pharmacol Biochem Behav 30: 737-748.

Carrera MR, Schulteis G, Koob GF (1999). Heroin self-administration in dependent Wistar rats: increased sensitivity to naloxone. Psychopharmacology 144: 111-120.

Carroll ME, Lac ST (1987). Cocaine withdrawal produces behavioral disruption in rats. Life Sci 40: 2183-2190.

Clark RD, Poling A (1990). Effects of cocaine administration and withdrawal on the performance of pigeons under a fixedconsecutive number schedule with and without an external discriminative stimulus. Behav Pharmacol 1: 491-496.

Czoty PW, Martelle JL, Nader MA (2006). Influence of abstinence and conditions of cocaine access on the reinforcing strength of cocaine in nonhuman primates. Drug Alcohol Depend 85: 213-220.

Czoty PW, McCabe C, Nader MA (2005). Assessment of the relative reinforcing strength of cocaine in socially housed monkeys using a choice procedure. J Pharmacol Exp Ther 312: 96-102.
Deneau G, Yanagita T, Seevers MH (1969). Self-administration of psychoactive substances by the monkey. Psychopharmacologia 16: $30-48$.

Gawin FH, Kleber HD (1986). Abstinence symptomatology and psychiatric diagnosis in cocaine abusers. Arch Gen Psychiatry 43: 107-113.

Glowa JR, Fantegrossi WE (1997). Effects of dopaminergic drugs on food- and cocaine-maintained responding. IV: Continuous cocaine infusions. Drug Alcohol Depend 45: 71-79.

Grabowski J, Rhoades H, Schmitz J, Stotts A, Daruzska LA, Creson $\mathrm{D}$ et al. (2001). Destroamphetamine for cocaine-dependence treatment: a double-blind randomized clinical trial. J Clin Psychopharmacol 21: 522-526.

Grabowski J, Rhoades H, Stotts A, Cowan K, Kopecky C, Dougherty A et al. (2004). Agonist-like or antagonist-like treatment for cocaine dependence with methadone for heroin dependence: two double-blind randomized clinical trials. Neuropsychopharmacology 29: 969-981.

Griffiths RR, Wurster RM, Brad (1975). Discrete-trial choice procedure: effects of naloxone and methadone on choice between food and heroin. Pharmacol Rev 27: 357-365.

Hammer Jr RP, Pires WS, markou A, Koob GF (1993). Withdrawal following cocaine self-administration decreases regional cerebral metabolic rate in critical brain reward regions. Synapse 14: $73-80$.

Kampman KM, Volpicelli JR, McGinnis DE, Alterman AI, Weinrieb RM, D'Angelo L et al. (1998). Reliability and validity of the cocaine selective severity assessment. Addict Behav 23: 449-461.

Kenny PJ, Polis I, Koob GF, Markou A (2003). Low dose cocaine self-administration transiently increases but high dose cocaine persistently decreases brain reward function in rats. Eur J Neurosci 17: 191-195.

Kleven MS, Woolverton WL (1991). Effects of continuous cocaine administration on schedule-controlled behavior in rhesus monkeys. Behav Pharmacol 2: 471-480.

Koob GF (2009). Neurobiological substrates from the dark side of compulsivity in addiction. Neuropharmacology 56(Suppl 1): $18-32$.

Kreek MJ, LaForge KS, Butelman E (2002). Pharmacotherapy of addictions. Nat Rev Drug Discov 1: 710-726.

Li DH, Depoortere RY, Emmett-Oglesby MW (1994). Tolerance to the reinforcing effects of cocaine in a progressive ratio paradigm. Psychopharmacology 116: 326-332.

Liu Y, Roberts DC, Morgan D (2005). Effects of extended-access self-administration and deprivation on breakpoints maintained by cocaine in rats. Psychopharmacology 179: 644-651.

Lowinson JH, Payte JT, Salsitz E, Joseph H, Marion IJ, Dole VP (1997). Methadone maintenance, In: Lowinson JH, Ruiz P, Millman RB, Langrod JG (eds). Substance Abuse: A Comprehensive Textbook. Williams and Wilkins: Baltimore. pp 405-425.

Malin DH, Moon WD, Moy ET, Jennings RE, Moy DM, Warner RL et al. (2000). A rodent model of cocaine abstinence syndrome. Pharmacol Biochem Behav 66: 323-328.

Markou A, Koob GF (1991). Postcocaine anhedonia. An animal model of cocaine withdrawal. Neuropsychopharmacology 4: $17-26$.

Mooney ME, Herin DV, Scmitz JM, Moukaddam N, Green CE, Grabowski J (2009). Effects of oral methamphetamine on cocaine use: a randomized, double-blind, placebo-controlled trial. Drug Alcohol Depend 101: 34-41.

Morgan D, Brebner K, Lynch WJ, Roberts DC (2002). Increases in the reinforcing efficacy of cocaine after particular histories of reinforcement. Behav Pharmacol 13: 389-396.

Morgan D, Roberts DC (2004). Sensitization to the reinforcing effects of cocaine following binge-abstinent self-administration. Neurosci Biobehav Rev 27: 803-812. 
Morgan D, Smith MA, Roberts DC (2005). Binge self-administration and deprivation produces sensitization to the reinforcing effects of cocaine in rats. Psychopharmacology 178: 309-316.

Nader MA, Woolverton WL (1992). Choice between cocaine and food by rhesus monkeys: effects of conditions of food availability. Behav Pharmacol 3: 635-638.

Negus SS (2003). Rapid assessment of choice between cocaine and food in rhesus monkeys: effects of environmental manipulations and treatment with D-amphetamine and flupenthixol. Neuropsychopharmacology 28: 919-931.

Negus SS (2004). Effects of the kappa opioid agonist U50,488 and the kappa opioid antagonist nor-binaltorphimine on choice between cocaine and food in rhesus monkeys. Psychopharmacology 176: 204-213.

Negus SS (2005a). Effects of punishment on choice between cocaine and food in rhesus monkeys. Psychopharmacology 181: 244-252.

Negus SS (2005b). Interactions between the reinforcing effects of cocaine and heroin in a drug-vs-food choice procedure in rhesus monkeys: a dose-addition analysis. Psychopharmaology 180: 115-124.

Negus SS (2006). Choice between heroin and food in nondependent and heroin-dependent rhesus monkeys: effects of naloxone, buprenorphine and methadone. J Pharmacol Exp Ther 317: 711-723.

Negus SS, Rice KC (2009). Mechanisms of withdrawal-associated increases in heroin self-administration: pharmacological modulation of heroin vs food choice in heroin-dependent rhesus monkeys. Neuropsychopharmacology 34: 899-911.

Orio L, Edwards S, George O, Parsons LH, Koob GF (2009). A role for the endocannabinoid system in the increased motivation for cocaine in extended-access conditions. J Neurosci 29: 4846-4857.

Panlilio LV, Goldberg SR, Gilman JP, Jufer R, Cone EJ, Schindler CW (1998). Effects of delivery rate and non-contingent infusion of cocaine on cocaine self-administration in rhesus monkeys. Psychopharmacology 137: 253-258.

Paronis CA, Gasior M, Bergman J (2002). Effects of cocaine under concurrent fixed ratio schedules of food and IV drug availability: a novel choice procedure in monkeys. Psychopharmacology 163 283-291.

Parsons LH, Koob GF, Weiss F (1995). Serotonin dysfunction in the nucleus accumbens of rats during withdrawal after unlimited access to intravenous cocaine. J Pharmacol Exp Ther 274: $1182-1191$

Paterson NE, Markou A (2003). Increased motivation for selfadministered cocaine after escalated cocaine intake. Neuroreport 14: 2229-2232.

Rhoades HM, Creson D, Elk R, Schmitz J, Grabowski J (1998). Retention, HIV risk, and illicit drug use during treatment: methadone dose and visit frequency. Am J Public Health 88: 34-39.

Siegel RK (1982). Cocaine smoking. J Psychoactive Drugs 14: 321-327.

Spragg SDS (1940). Morphine addiction in chimpanzees. Comp Psychol Monogr 15: 5-132.

Stitzer M, Petry N (2006). Contingency management for treatment of substance abuse. Annu Rev Clin Psychol 2: 411-434.

Vocci FJ, Acri J, Elkashef A (2005). Medication development of addictive disorders: the state of the science. Am J Psychiatry 162: $1432-1440$

Wee S, Mandyam CD, Lekic DM, Koob GF (2008). alpha-1 noradrenergic system role in increased motivation for cocaine intake in rats with prolonged access. Eur Neuropsychopharmacol 18: 303-311.

Weiss F, Markou A, Lorang MT, Koob GF (1992). Basal extracellular dopamine levels in the nucleus accumbens are decreased during cocaine withdrawal after unlimited-access selfadministration. Brain Res 593: 314-318.

Woolverton WL, Kleven MS (1988). Evidence for cocaine dependence in monkeys following prolonged periods of exposure. Psychopharmacology 94: 288-291.

Yanagita T (1975). Some methodological problems in assessing dependence-producing properties of drugs in animals. Pharmacol Rev 27: 503-509.

Yanagita T (1978). Drug dependence studies in laboratory animals. NIDA Res Monogr 19: 179-190.

Yanagita T (1980). Self-administration studies on psychological dependence. Trends Pharmacol Sci 1: 161-164. 